\title{
Risks and benefits of prophylactic antimalarial drugs
}

\author{
Chloroquine, by itself or with proguanil, or mefloquine should be used according to circumstances
}

In recent years up to 2000 cases of malaria have been reported annually in Britain. Worryingly, an increasing proportion (about half) are due to infection with Plasmodium falciparum, and some $1 \%$ of these patients die. ${ }^{1}$ The main efforts of malaria prophylaxis are against $P$ falciparum; infection with $P$ vivax, $P$ ovale, and $P$ malariae may cause severe morbidity but rarely causes death. These numbers of cases of malaria have increased dramatically over the past 10 years for several reasons. Firstly, we are becoming increasingly adventurous and frequent travellers on both holiday and business. Secondly, when we seek advice on prophylactic measures the advice is often of poor quality and contradictory, and in any case we do not often comply with it. Thirdly, the emergence of resistance of $P$ falciparum, not only to chloroquine but also to all other available antimalarial drugs in South America, East and West Africa, and many parts of Asia has meant a decline in the efficacy of formerly useful agents. Fourthly, there are increasing numbers of reports of serious adverse effects with the various substitute drugs proposed.

Though no single agent or drug combination can guarantee safe and effective protection against malaria, drugs still play an extremely important part in prophylaxis. Thus practitioners whose advice is sought by their travelling patients must be able to avail themselves of information on regional variations in transmission rates of malaria and regional patterns of resistance to antimalarial drugs from local community medicine specialists with access to authoritative information such as from schools of tropical medicine. They must also be able to assess the risks and benefits of the antimalarial prophylactic drugs they will prescribe.

Unfortunately, the databases from which sound prophylactic advice can be given are frequently inadequate. Advice must be simple and clear, must encourage good compliance, must provide reasonable protection against malaria, must cause minimal risk of adverse effects, and should apply to most people. ${ }^{2}$ Any adverse effects encountered with antimalarial drugs should be reported on "yellow cards", thus building up our information on this increasingly difficult subject.

Few drugs - chloroquine, proguanil, mefloquine hydrochloride, and Maloprim (a combination of pyrimethamine and dapsone) - are now used for malaria prophylaxis. Fansidar (a combination of pyrimethamine and sulphadoxine) and amodiaquine are no longer recommended because of the nature, frequency, and severity of the adverse reactions produced.
Few problems are encountered with chloroquine at the doses prescribed for malaria prophylaxis (300 mg weekly), and it is one of the oldest drugs in our therapeutic armament. Transient gastrointestinal discomfort is not unusual, and diarrhoea may occur. In black patients pruritus is common, but the reasons for this are not clear. Taking chloroquine for several years may lead to retinopathy, but this is usually encountered when the drug is given at higher doses for rheumatoid arthritis and not in the usual doses and for the usual duration of malarial prophylaxis.

Proguanil is probably the safest of the current antimalarial drugs. A biguanide, proguanil itself is inactive and requires metabolism to cycloguanil, which inhibits selectively malarial dihydrofolate reductase activity. People vary in their rate of metabolic activation of proguanil, but the clinical implications of this are not clear. ${ }^{3}$ Patients taking proguanil may develop mouth ulcers, abdominal discomfort, and vomiting, but these effects are not usually serious. Alopecia has also been recorded, as has megaloblastic anaemia in patients with kidney disease.

Mefloquine hydrochloride has been introduced recently as an antimalarial drug and is given for prophylaxis in a dose of $250 \mathrm{mg}$ once weekly. Unfortunately, resistance of $P$ falciparum has already been recorded and is a cause for great concern. Nausea and abdominal discomfort, vomiting, and to a lesser extent diarrhoea are all common adverse effects found soon after taking the drug, and dizziness, disturbance of balance, and confusion - in some cases amounting to psychotic disturbance $^{45}$ - are less common. Their relation to dose (and plasma concentrations) of mefloquine hydrochloride are not clear, nor is their cause. ${ }^{6}$

Maloprim (12.5 mg pyrimethamine and $100 \mathrm{mg}$ dapsone) should now be prescribed only rarely (see below): agranulocytosis is too frequently found for its widespread use (one in 10000 prescriptions). Other authors have documented a prevalence of agranulocytosis as high as one in 2000 if the recommended dose of one tablet a week is exceeded. ${ }^{7}$ Less seriously, methaemoglobinaemia and megaloblastic anaemia have also been described. Other drugs whose use in malaria prophylaxis has been superseded are Fansidar, because of the frequency of blood dyscrasias, skin reactions, and the Stevens Johnson syndrome, and amodiaquine, because of agranulocytosis and liver damage.

How then should doctors use this information in advising their patients? Chloroquine is the drug of choice for the traveller to those countries where $P$ vivax is predominant and 
the decreasing number of areas where $P$ falciparum is still sensitive to the drug. Even in areas where $P$ falciparum is known to be resistant to chloroquine many parasites remain sensitive to the drug. There the advice currently is either to combine proguanil $200 \mathrm{mg}$ daily with chloroquine $300 \mathrm{mg}$ once a week or to use mefloquine hydrochloride. When short term exposure (three weeks or less) is envisaged in areas where chloroquine resistance is widespread, mefloquine hydrochloride may now be the treatment of choice and is currently being evaluated. The use of Maloprim is restricted to travellers to Papua New Guinea, the Solomon Islands, and the Southern Pacific, where good results have been reported in Australian travellers, although haematological monitoring is necessary.

When drugs are given for malarial prophylaxis they should always be started at least one week before travel to test for any idiosyncratic reaction and to attain steady state plasma concentrations. They must also be continued for four weeks after leaving the malarious area. Personal protection, such as wearing clothes with long sleeves and trousers in the evening, using insect repellents, and sleeping under intact mosquito nets, is of the highest importance where drug resistance is common. Special cases such as the pregnant traveller and the immunocompromised patient demand expert advice.

Finally, as the late Brian Maegraith emphasised many years ago in his classic article Unde venis, if the traveller develops a fever or feels unwell on returning from a malarious area, irrespective of the prophylactic measures that have been adopted, the possibility of malarial infection must always be seriously considered and correctly managed. ${ }^{8}$

ALASDAIR BRECKENRIDGE

Professor of Clinical Pharmacology,

University of Liverpool,

Liverpool L69 3BX

1 Gilles HG. Malaria - an overview. I Infect 1987;18:11-23.

2 Phillips-Howard PA, Blaze M, Burn M, Bradley DJ. Malarial prophylaxis: survey of the response of British travellers to prophylactic advice. Br Med f 1986;293:932-4.

3 Ward SA, Watkins WM, Mberu E, et al. Inter-subject variability in the metabolism of proguanil to the active metabolite cycloguanil in man. Br f Clin Pharmacol 1989;27:781-7.

4 Stuiver PC, Ligthelm RJ, Goud TJLM. Acute psychosis after mefloquine. Lancet 1989;ii:282.

5 Björkman A. Acute psychosis following mefloquine prophylaxis. Lancet 1989;ii:865.

6 UNDP, World Bank WHO Update. Development of mefloquine as an antimalarial drug. Bull WHO 1983;61:169-78.

7 Friman G, Nyström-Rosander C, Jonsell G, Björkman A, Lekăs G, Svendrưp B. Agranulocytosis assaciated with malaria prophylaxis with Maloprim. Br Med 7 1983;286:1244-5.

8 Maegraith B. Unde venis? Lancet 1963;i:401-4.

\title{
Survival of patients with cancer
}

\author{
Those included in clinical trials do better
}

Discussion and argument continue about where patients with cancer should be treated and the place of standardised protocols and clinical trials. ${ }^{1-4}$ General conclusions are hard to draw as most published data have been on the rarer cancers, many of them occurring in childhood, for which the most impressive advances in treatment have been made. The topic should be taken a little further with the publication ( $p$ 1069) of a large population based study in Finland of the effects of treatment protocols on survival for multiple myeloma, an adult cancer which still has a poor prognosis.

Some of the earliest evidence of the effect of inclusion in trials comes from studies of acute lymphoblastic leukaemia in England and Wales during the 1960s. Survival then was very low, but children with acute lymphoblastic leukaemia survived longer if they were treated by physicians specialising in this disease. Since then there have been great improvements in survival, in which clinical trials have played an important part $^{6}$ : children with acute lymphoblastic leukaemia diagnosed and treated in Britain throughout 1971-84 had a higher survival rate if they were included in the Medical Research Council trials. ${ }^{7}$ For children in the trials the size of the treatment centre (measured by number of new child patients with acute lymphoblastic leukaemia per year) had no effect on survival, but among patients not in the trials survival was higher at hospitals treating larger numbers. Substantial advantages of treatment according to protocol and at specialist centres were also found for children with acute lymphoblastic leukaemia in 1970-5 in the Greater Delaware Valley in the United States. ${ }^{\times}$In the British series there was considerable variation in survival among major centres. Some of this variation was probably attributable to differences in the distribution of prognostic factors, but some may also have been due to differences in the degree of compliance with standard protocols. ${ }^{4}$ For acute non-lymphoblastic leukaemia the outlook is still`poor, but during 1977-84 children treated in specialist paediatric units and teaching hospitals had a higher survival rate. ${ }^{\prime \prime}$

Similar patterns have been found for several other childhood cancers. Retinoblastoma has long had an excellent prognosis, but patients treated at specialist centres have a consistently higher survival rate." ${ }^{12}$ Children with Wilms' tumour in the first Medical Research Council trial had a higher survival rate than those who were eligible and not included. ${ }^{13}$ Since the formation of the United Kingdom Children's Cancer Study Group in 1977 the care of children with cancer has steadily become more centralised, and nowadays more than two thirds of patients are treated by group members. Survival was higher at paediatric oncology centres than elsewhere for children with non-Hodgkin's lymphoma. Ewing's tumour, and rhabdomyosarcoma treated during 1977-84 and osteosarcoma during 1981-4. ${ }^{10}$ No differences were found for Hodgkin's disease, Wilms' tumour or neuroblastoma, but the paediatric centres had a considerably higher proportion of patients with advanced neuroblastoma. There is evidence that some children with Wilms' tumour treated at other hospitals and not included in the United Kingdom Children's Cancer Study Group clinical studies were overtreated ${ }^{1+}$; similarly, in the Greater Delaware Valley survivors at non-specialist hospitals had a less thorough follow up for the late effects of treatment. ${ }^{15}$

By contrast, few comparative studies have been done for adult cancers. A study of osteosarcoma in patients under 65 in the south Thames regions showed little benefit of treatment at centres with the greatest experience of this disease, ${ }^{16}$ but doubts were expressed over the heterogeneity of patients in the series, ${ }^{17}$ and improved results in younger patients at specialist centres may have been too recent to be recognised in this analysis. ${ }^{18}$ For multiple myeloma survival has now been shown to be higher for patients resident in districts with a policy of entry to clinical trials (p 1069). The prognosis for 